\title{
SOCIAL NETWORKING WEBSITES FROM THE POINT OF VIEW OF UNIVERSITY STUDENTS: A COMPARATIVE ANALYSIS
}

\author{
Vincentas Lamanauskas, Violeta Šlekienè, Loreta Ragulienè \\ University of Siauliai, Lithuania \\ E-mail: v.lamanauskas@ef.su.It, violeta@fm.su.It, loretar@gmail.com
}

Dragos Iordache, Costin Pribeanu

National Institute for Research and Development

in Informatics - ICI Bucharest, Romania

E-mail: pribeanu@ici.ro

Martin Bilek
University of Hradec Kralove, Czechia
E-mail: martin.bilek@uhk.cz

Bulent Cavas

Dokuz Eylul University, Turkey

E-mail: bulent.cavas@deu.edu.tr

Tatyana Mazurok

South Ukrainian National Pedagogical University named after K.D. Ushynsky, Ukraine

E-mail: pdpu@pdpu.edu.ua

\begin{abstract}
The use of social networking websites is becoming increasingly popular. While there are many reasons for this, the rapid spread of ICT is one of the most determining factors. New technologies provide various possibilities for education and in relation to social networking websites it is worth emphasizing their diverse possibilities. The functionality of social networking websites is increasing, and this, in its own way, opens many possibilities for the consumers, a factor encouraging the usage of social networking sites. This research relates to the usage of social networking websites. The purpose of the research is to ascertain how university students from a range of European countries (Lithuania, Romania, Ukraine, Czech Republic, Turkey) use social networking websites; how they value them; what opinion they have about various social networking websites; what they know about them.

A questionnaire, Social Networks, with 16 questions was used in the research. The breakdown of the questions is as follows: 3 questions were allotted to establishing the characteristics of the respondents and 5 open ended type questions were to possible advantages and disadvantages of SNW. The $6^{\text {th }}$ question was assigned to evaluate the frequency of social networking website usage. A list of 23 main networking websites was presented. The $8^{\text {th }}$ question was to evaluate the functions of social networking websites (e.g., communication, advertisement, friend search, information exchange and so on). In addition, the questionnaire also presented 26 various type statements about social networking websites and respondents were asked to evaluate these by applying the interval Likert scale (from "Completely agree" to "Completely disagree"). The original questionnaire was translated into the national languages by the local researchers involved in the study.
\end{abstract}


Vincentas LAMANAUSKAS, Violeta ŠLEKIENĖ, Loreta RAGULIENĖ, Dragos IORDACHE, Costin PRIBEANU, Martin BILEK, Bulent CAVAS, Tatyana MAZUROK. Social Networking Websites From the Point of View of University Students: a Comparative Analysis

\section{PROBLEMS \\ OF EDUCATION \\ IN THE $21^{\text {st }}$ CENTURY Volume 57,2013

This education research was carried out in 2012-2013, recognizing that it is especially important to understand the essential motives of using social networking websites, to analyze their probable advantages and disadvantages, to make some comparisons among students from the different countries.

The findings illustrated that the respondents knew a lot of social networking websites, with the most frequently visited and most popular being: You Tube, Facebook and Google+. The most important functions put forward by the university students were: communication, learning and exchanging information, texting. Gender differences were noted in the case of valuing social networking websites.

The university students pointed out the following $S N$ website advantages:

(a) the possibility to communicate with many people at one time,

(b) to get in touch with the people seen long ago also with the friends and relatives living abroad,

(c) the possibility to find proper information and share it, etc.

The respondents also noted that $S N$ website had disadvantages. They indicated: lack of information publicity, a lack of privacy, insecurity in presented data, possible lies, deceptions, etc.

Key words: comparative analysis, ICT, social networking websites, university students.

\section{Introduction}

It is obvious, that usage of social networking websites is constantly growing. An evident tendency is the expansion of SNW possibilities in a business environment. If earlier it was thought that usage of SNW is a waste of time, in the latter years business finds more and more reasons why social networking websites ought to be used. This is marketing, service and product advertisement, new client search, cooperation development and so on. The other thing - the amount and the flow of information will certainly not decrease and therefore information usage and management in general, will remain urgent.

The other tendency is also rather distinct. Social networking websites "move" into educational space as well. However, a great number of problems exist here. Nowadays teachers (speaking especially about Lithuanian situation) are not properly prepared in ICT field. Though ICT usage competences are being improved in general, however considerable gaps remain, the shortage of understanding is felt on how to apply information technologies, what their abilities are to make teaching/learning process easier, more effective and interesting for children. ICT in its own way changes rapidly, it is complicated to adjust to existing technological environment. On the other hand, nowadays children have grown up in ICT surroundings, in the environment more or less filled with technologies. This makes them more susceptible to technologies in general. Thus, teachers' society can't stay behind from ICT development either. Teachers themselves have to be ready for unpredictable future (or at least hardly predictable) and to convey this attitude to children. A continuous demand to improve becomes the cornerstone for every member of society.

The problem of motivation to learn and improve remains urgent. Despite education science achievements, motivation encouragement problem remains one of the most serious ones. However, watching children and teenagers' activities using ICT, especially social networking websites, a very high motivation, a demand to use SNW are fixated. Thus, it is important to use up that motivation in educational sense, in other words, to use up social networking websites abilities in educational sense. It is already a common and understandable thing in itself to speak about higher technologies in industry, medicine, pharmacy, agriculture and so on. Somehow it is spoken very little or not spoken at all about higher technologies in education (pedagogy of higher technology).

Thus, the discussed field remains problematic. New, exhaustive, empiric researches are necessary. The former researches (Lamanauskas and other, 2010) showed, that students have unlimited access to the internet, a possibility to use broadband (cable), a phone line (modem) or wireless internet. Therefore, it is not a surprise, that such a big number of the respondents use the internet even a few times a day. The mentioned research also showed, that earlier researches (Lamanauskas and other, 2010) showed, that students use the internet not only for communica- 
tion (usage index $\mathrm{UI}=0.92, \mathrm{SD}=0.21)$, but also for general information search $(\mathrm{UI}=0.91, \mathrm{SD}$ $=0.19$ ), for $\mathrm{e}-$ mail services $(\mathrm{UI}=0.86, \mathrm{SD}=0.26)$, for listening to music $(\mathrm{UI}=0.84, \mathrm{SD}=$ $0.28)$, for studies $(\mathrm{UI}=0.81, \mathrm{SD}=0.26)$ and other. It is natural, that speaking about social networking websites, almost the biggest attention is paid to Facebook. A. Joinson (2008) having carried out a research, discerned seven unique uses and gratifications: social connection, shared identities, content, social investigation, social network surfing and status updating. Ellison et al (2007) studied the relationship between Facebook use and the formation and maintenance of social capital. Putnam (2000) distinguishes between bridging (weak ties) and bonding (close relationships) social capital. In a more recent work, Jung et al (2013) unpacked the dimensions of social capital and found out that some sub-dimensions play an important role in getting favors from Facebook friends while the two dimensions (bridging and bonding) do not significantly predict getting favors. As the researchers claim, Facebook offers a lot of opportunities for developers and educators too (Awouters, Palmaers, Veltjen, 2011).

Problems lie not only in earlier discussed things. Privacy questions are very important (Acquisti, Gross, 2005; Kolek \& Saunders, 2008), and on the whole, social behavior, habits, technology usage (Gemmill, Peterson, 2006) and other questions. Jesse P. Mendez, J. Curry, M. Mwavita, K. Kennedy, K. Weinland, K. Bainbridge (2009) investigated academic interaction problems in social networking websites. They established that students are using social networking websites rather productively, however mostly with the social communication purposes. In other words, a strategy remains to create personal relations and to develop them in virtual internet world, communicating through internet social networking websites. The usage of the newest technologies is rapidly growing, though such growth is rather different in various countries. For example, in Japan both teachers and lecturers are trying to use as much as possible of technologies in their direct work (Taynton, 2012), however, it remains unclear how students perceive themselves as users of technology, the types of use of that technology and their perceptions. Academic achievement question is urgent. Rouis, S., Limayem, M., SalehiSangari, E. (2011) ascertained, that the usage of social networking websites makes the academic students' achievements worse. He states, that personality traits and self-regulation significantly determine the use of Facebook. However, Facebook use negatively affects students` academic performance. The research, carried out in Czech republic also showed, that SNW users are interested more in social parameters of such networking websites' usage (e.g. to make friends, to establish new relationships and other) than academic question or problem solution (e.g., to obtain missing information, advice, recommendation and other) (Karliček, Tomek, Hasprova, Zamazalova, 2012). Regardless doubts, social networking websites have plenty of unrevealed educational possibilities.

Regardless of clearly common tendencies, it can be stated, that in different countries certain differences can be observed. The research, carried out in Romania showed, that the motivations that determine young people to use online social networks are psychological ones, and they are strongly related to the human needs of communication, socialization, being an active part of a group, or maintaining long distance friendships (Baltaretu, Balaban, 2010). A qualitative research carried out later, showed similar motives, in fact, because the most important things for the consumers are: the idea of belonging as a member to a social network site is associated to the desire of integrating and being accepted in a group as equal, to the desire of not losing contact with friends, to the appropriate medium for setting up a new friendship and at the same time, relaxing, and having a great free time (Marinescu, 2011). One of the newest researches, carried out in Turkey shows, that most of the students did not trust virtual friendships, and did not like sharing photographs and political views online (Turan, Tinmaz, Goktas, 2013). On the other hand, research showed, that students ' duration of use of social networks varies according to the type of foundation or state university, they do not prefer social networks for discovery and recognition of themselves and their peers etc. (Ünal Tutgun, Köroğlu, 2013).

Thus, research object is the usage of social networking websites. Research purpose is to ascertain how 5 countries (Lithuania, Romania, Czech Republic, Turkey, Ukraine) uni-

\author{
PROBLEMS \\ OF EDUCATION \\ IN THE $21^{\text {st }}$ CENTURY \\ 63
}


Vincentas LAMANAUSKAS, Violeta ŠLEKIENĖ, Loreta RAGULIENĖ, Dragos IORDACHE, Costin PRIBEANU, Martin BILEK, Bulent CAVAS, Tatyana MAZUROK. Social Networking Websites From the Point of View of University Students: a Comparative Analysis

OF EDUCA

IN THE $21^{\text {st }}$ CENTURY Volume 57,2013

64 versity students use social networking websites, how they value them, what opinion they have about various social networking websites, what they know about them. The main research questions are:

- What social networking websites do students know and use most frequently?

- What social networking website functions do students use and why?

- Do students like social networking websites and why?

- What do students know about how social networking websites use published personal and other type of information?

- What is students' attitude to possibilities provided by social networking websites?

\section{Methodology of Research}

\section{General Research Characteristics, Respondents}

In 2012-2013, 2058 1-st to 5-th course university study students from 5 countries participated in the research (table 1). 918/44.6\% of them from Lithuania, 709/34.4\% - from Czech Republic, 172/8.4\% - from Romania, 142/6.9\% - from Ukraine and 117/5.7\% from Turkey.

Table 1. Information about the respondents ( $\mathrm{N} / \%)$.

\begin{tabular}{lllllll}
\hline \multirow{2}{*}{ According to country } & $\begin{array}{l}\text { Lithuania } \\
\text { (LT) }\end{array}$ & $\begin{array}{l}\text { Romania } \\
\text { (RO) }\end{array}$ & $\begin{array}{l}\text { Ukraine } \\
\text { (UA) }\end{array}$ & Turkey (TR) & $\begin{array}{l}\text { Czech Republic } \\
\text { (CZ) }\end{array}$ & Total \\
\cline { 2 - 7 } & $918 / 44.6$ & $172 / 8.4$ & $142 / 6.9$ & $117 / 5.7$ & $709 / 34.4$ & $2058 / 100$ \\
\hline
\end{tabular}

\section{Instrument}

The authors' prepared questionnaire was used in the research. 16 questions form the questionnaire Social networks. 3 questions in the questionnaire are allotted to establish the characteristics of the respondents, 5 open type questions. The $6^{\text {th }}$ question was assigned to evaluate the frequency of social networking website usage. 23 main networking websites are presented in the list. The $8^{\text {th }}$ question was allotted to evaluate the functions of social networking websites (e.g., communication, advertisement, friend search, information exchange and so on). Also, 26 various type statements about social networking websites were presented and respondents were asked to evaluate them applying the interval Likert scale (from "Completely agree" to "Completely disagree").

\section{Statistical Data Analysis}

In order to analyze research data, measures of descriptive statistics are applied (absolute and relative frequencies, popularity or usage indexes $(0 \leq \mathrm{PI} / \mathrm{UI} \leq 1)$, standard deviations. The closer is PI/UI value to 1 , the more important, more significant is the statement to the respondent, or respondent better approves of it. The SSPS statistics batch is used as an instrument for data processing.

\section{Research Results}

The respondents were asked about using the internet and participating in social networking sites. The analysis of the question How often do you use the internet? showed, that from all respondents, even 1774/86.2\% of students use the internet a few times a day (table 3), 195/9.5\% - once a day, 49/2.4\% - 3-5 times per week, and a very small part of respondents 20/1.0\% - 1-2 times per week. Analysing according to countries, one can see that mostly Ukrai- 
Vincentas LAMANAUSKAS, Violeta ŠLEKIENĖ, Loreta RAGULIENĖ, Dragos IORDACHE, Costin PRIBEANU, Martin BILEK, Bulent CAVAS, Tatyana MAZUROK. Social Networking Websites From the Point of View of University Students: a Comparative Analysis

ne respondents $(92.3 \%)$ use the internet a few times a day. Though Turkish students, as well, mostly use the internet a few times a day (78.6\%), however, even $17.9 \%$ go on the internet once a day. Only $13 / 0.6 \%$ of all country respondents use the internet every two weeks or less. It can be said, that nowadays youth cannot imagine their day without the internet.

Table 2. The frequency of using the internet (N/\%). N = 2058

\begin{tabular}{lllllll}
\hline Usage frequency & LT & RO & UA & TR & CZ & $\begin{array}{l}\text { All coun- } \\
\text { tries }\end{array}$ \\
\hline A few times a day & $795 / 86.6$ & $144 / 83.7$ & $131 / 92.3$ & $92 / 78.6$ & $612 / 86.3$ & $1774 / 86.2$ \\
\hline Once a day & $81 / 8.8$ & $20 / 11.6$ & $9 / 6.3$ & $21 / 17.9$ & $64 / 9.0$ & $195 / 9.5$ \\
\hline 3-5 times per week & $26 / 2.8$ & $4 / 0.6$ & $1 / 0.7$ & $0 / 0$ & $18 / 2.5$ & $49 / 2.4$ \\
\hline 1-2 times per week & $12 / 1.3$ & $4 / 0.6$ & $0 / 0$ & $1 / 0.85$ & $3 / 0.4$ & $20 / 1.0$ \\
\hline Every two weeks & $3 / 0.3$ & $0 / 0$ & $1 / 0.7$ & $2 / 1.7$ & $5 / 0.7$ & $11 / 0.5$ \\
\hline Less & $0 / 0$ & $0 / 0$ & $0 / 0$ & $1 / 0.85$ & $1 / 0.14$ & $2 / 0.1$ \\
\hline Missing & $1 / 0.1$ & $0 / 0$ & $0 / 0$ & $0 / 0$ & $6 / 0.85$ & $7 / 0.3$ \\
\hline Total & $918 / 100$ & $172 / 100$ & $142 / 100$ & $117 / 100$ & $709 / 100$ & $2058 / 100$ \\
\hline
\end{tabular}

It was compared, how much time on avarage students spend on the internet during one visit. It appears, that during one visit on the internet the respondents mostly spend from half an hour to two hours (table 3): between 10 to $30 \mathrm{~min}$. $493 / 24.0 \%$, between $30 \mathrm{~min}$. to $1 \mathrm{~h}$. $534 / 25.9 \%$, between 1 to $2 \mathrm{~h}$. $-459 / 22.3 \%$. $261 / 12.7 \%$ of the respondents spend more than 3 hours on the internet. $32.3 \%$ of Czech respondents spend from 10 to 30 min. during one visit on the internet and $29.6 \%$ of Ukraine respondents - even more than 3 hours.

Table 3. Students about the time spent on the internet (N/\%). N = 2058

\begin{tabular}{lllllll}
\hline Time & LT & RO & UA & TR & CZ & All countries \\
\hline Less than 10 min. & $16 / 1.7$ & $7 / 4.1$ & $4 / 2.8$ & $5 / 4.3$ & $30 / 4.2$ & $62 / 3.0$ \\
\hline From10 to 30 min. & $202 / 22.0$ & $25 / 14.5$ & $14 / 9.9$ & $23 / 19.7$ & $229 / 32.3$ & $493 / 24.0$ \\
\hline From 30 min. to 1h. & $237 / 25.8$ & $46 / 26.7$ & $33 / 23.2$ & $27 / 23.1$ & $191 / 26.9$ & $534 / 25.9$ \\
\hline From 1 to 2h. & $242 / 26.4$ & $41 / 23.8$ & $35 / 24.6$ & $31 / 26.5$ & $110 / 15.5$ & $459 / 22.3$ \\
\hline From 2 to 3 h. & $118 / 12.9$ & $24 / 14.0$ & $14 / 9.9$ & $10 / 8.5$ & $70 / 9.9$ & $236 / 11.5$ \\
\hline More than 3 h. & $101 / 11.0$ & $29 / 16.9$ & $42 / 29.6$ & $21 / 17.9$ & $68 / 9.6$ & $261 / 12.7$ \\
\hline Missing & $2 / 0.2$ & $0 / 0$ & $0 / 0$ & $0 / 0$ & $11 / 1.6$ & $13 / 0.6$ \\
\hline Total & $918 / 100$ & $172 / 100$ & $142 / 100$ & $117 / 100$ & $709 / 100$ & $2018 / 100$ \\
\hline
\end{tabular}

The question „How often do students visit social networking websites?“ analysis showed, that $1037 / 50.4 \%$ of respondents (table 4 ) visit online social networking websites at least a few times a day, $494 / 24.0 \%$ - at least once a day. A small part of them visit a few times per week, and $122 / 5.9 \%$ - less than every two weeks. Even $73.9 \%$ of Ukraine respondents visit 
Vincentas LAMANAUSKAS, Violeta ŠLEKIENĖ, Loreta RAGULIENĖ, Dragos IORDACHE, Costin PRIBEANU, Martin BILEK, Bulent CAVAS, Tatyana MAZUROK. Social Networking Websites From the Point of View of University Students: a Comparative Analysis

PROBLEMS

OF EDUCATION

IN THE $21^{\text {st }}$ CENTURY Volume 57,2013

social networking websites a few times a day and only $17.6 \%$ - once a day, and the rest $8.4 \%$ of respondents - a few times per week or less. Comparing with the usage of the internet, one can think, that some of the respondents haven't created a personal profile in social networking websites, however, they use the internet.

Table 4. The frequency of visiting social networking websites $(\mathrm{N} / \%) . \mathrm{N}=2058$

\begin{tabular}{lllllll}
\hline Visiting frequency & LT & RO & UA & TR & CZ & All countries \\
\hline A few times a day & $490 / 53.4$ & $71 / 41.3$ & $105 / 73.9$ & & $371 / 52.3$ & $1037 / 50.4$ \\
\hline Once a day & $240 / 26.1$ & $51 / 29.7$ & $25 / 17.6$ & & $178 / 25.1$ & $494 / 24.0$ \\
\hline 3-5 times per week & $34 / 3.7$ & $9 / 5.2$ & $9 / 6.3$ & & $31 / 4.4$ & $83 / 4.0$ \\
\hline 1-2 times per week & $53 / 5.8$ & $30 / 17.4$ & $1 / 0.7$ & & $32 / 4.5$ & $116 / 5.6$ \\
\hline Every two weeks & $17 / 1.9$ & $3 / 1.7$ & $1 / 0.7$ & & $12 / 1.7$ & $33 / 1.6$ \\
\hline Less & $59 / 6.4$ & $8 / 4.7$ & $1 / 0.7$ & & $54 / 7.6$ & $122 / 5.9$ \\
\hline Missing & $25 / 2.7$ & $0 / 0$ & $0 / 0$ & $117 / 100$ & $31 / 4.4$ & $173 / 8.4$ \\
\hline Total & $918 / 100$ & $172 / 100$ & $142 / 100$ & $117 / 100$ & $709 / 100$ & $2058 / 100$ \\
\hline
\end{tabular}

To the question How much time on avarage do you spend on the internet social networking websites during one visit ?- 723/35.1\% of the respondents answered, that they spend between $10 \mathrm{~min}$. and half an hour, and 447/21.7\% - less than 10min. (table 5). Most of the Ukraine respondents $(26.1 \%)$ spend from $30 \mathrm{~min}$. to $1 \mathrm{~h}$. on social networking websites during one visit, and $11.3 \%$ - stay even more than 3 hours. Comparing with the time spent on the internet in general, one can claim, that social networking websites occupy not a small part of the time spent on the internet. Turkish respondents did not give answers to these questions.

Table 5. Students about the time being spent on the social networking websites $(N / \%) \cdot N=2058$

\begin{tabular}{lllllll}
\hline $\begin{array}{l}\text { Average time being spent } \\
\text { during one visit }\end{array}$ & LT & RO & UA & TR & CZ & All countries \\
\hline Less than 10 min. & $191 / 20.8$ & $34 / 19.8$ & $15 / 10.6$ & $207 / 29.2$ & $447 / 21.7$ \\
\hline From10 to 30 min. & $377 / 41.1$ & $59 / 34.3$ & $35 / 24.6$ & $252 / 35.5$ & $723 / 35.1$ \\
\hline From 30 min. to $\mathrm{h}$. & $151 / 16.4$ & $33 / 19.2$ & $37 / 26.1$ & & $99 / 14.0$ & $320 / 15.5$ \\
\hline From 1 to 2h. & $113 / 12.3$ & $22 / 12.8$ & $28 / 19.7$ & $65 / 9.2$ & $228 / 11.1$ \\
\hline From 2 to 3 $\mathrm{h}$. & $43 / 4.7$ & $15 / 8.7$ & $11 / 7.7$ & & $27 / 3.8$ & $96 / 4.7$ \\
\hline More than 3 h. & $28 / 3.05$ & $9 / 5.2$ & $16 / 11.3$ & & $27 / 3.8$ & $80 / 3.9$ \\
\hline Missing & $15 / 1.6$ & $0 / 0$ & $0 / 0$ & $117 / 100$ & $32 / 4.5$ & $164 / 8.0$ \\
\hline Total & $918 / 100$ & $172 / 100$ & $142 / 100$ & $117 / 100$ & $709 / 100$ & $2058 / 100$ \\
\hline
\end{tabular}

Students were asked if they like social networking websites. Data analysis showed (table $6)$, that social networking websites are popular among youth $(\mathrm{PI}=0.62, \mathrm{SD}=0.33)$. 
Vincentas LAMANAUSKAS, Violeta ŠLEKIENĖ, Loreta RAGULIENĖ, Dragos IORDACHE, Costin PRIBEANU, Martin BILEK, Bulent CAVAS, Tatyana MAZUROK. Social Networking Websites From the Point of View of University Students: a Comparative Analysis

Table 6. Attitude to social networking websites. (PI - popularity index, $0 \leq \mathrm{PI} \leq 1$; SD - standard deviation)

\begin{tabular}{lllllll}
\hline $\begin{array}{l}\text { Do you like social network- } \\
\text { ing websites? }\end{array}$ & $\mathrm{LT}$ & $\mathrm{RO}$ & $\mathrm{UA}$ & $\mathrm{TR}$ & $\mathrm{CZ}$ & Total \\
& $\mathrm{PI} / \mathrm{SD}$ & $\mathrm{PI} / \mathrm{SD}$ & $\mathrm{PI} / \mathrm{SD}$ & $\mathrm{PI} / \mathrm{SD}$ & $\mathrm{PI} / \mathrm{SD}$ & $\mathrm{PI} / \mathrm{SD}$ \\
\hline & $0.66 / 0.30$ & $0.67 / 0.32$ & $0.65 / 0.28$ & $0.73 / 0.31$ & $0.54 / 0.35$ & $0.62 / 0.33$ \\
\hline
\end{tabular}

$\mathrm{SN}$ are most popular among Turkish students $(\mathrm{PI}=0.73, \mathrm{SD}=0.31)$ and a little less popular among Czech students ( $\mathrm{PI}=0.54, \mathrm{SD}=0.35$ ). The other country respondents' $\mathrm{PI}(\mathrm{LT}, \mathrm{RO}$, UA) are very close to average. Having analysed opinion distribution, one can see (Figure 1), that Czech and Lithuanian respondent evaluation has the least deviation from average. Turkish respondents ' popularity index is the highest, however, their opinion distribution is the biggest of all.

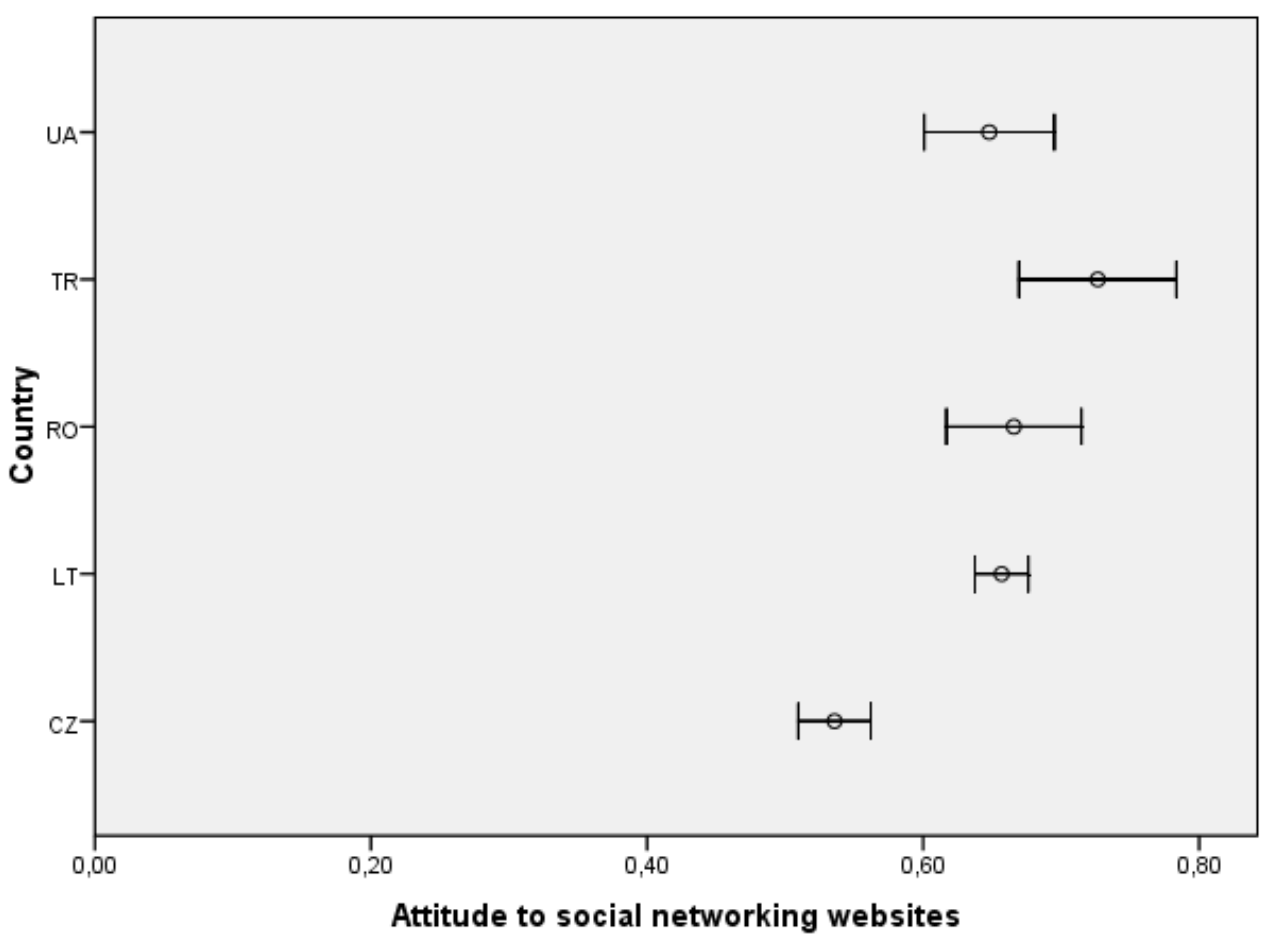

Error Bars: $95 \% \mathrm{Cl}$

Figure 1: Evaluation of social networking websites.

23 social networking website names were presented in the questionnaire and it was interesting to know, how often the respondents take part in them. Having made a generalization, one can put forward 3 main social networking websites (table 7), which are mostly visited by the respondents of the countries involved in the research. The most visited online social networks are: Facebook $(\mathrm{PI}=0.82, \mathrm{SD}=0.35)$, YouTube $(\mathrm{PI}=0.82, \mathrm{SD}=0.27)$ and Google+ $(\mathrm{PI}$ $=0.56, \mathrm{SD}=0.44)$. In these internet sites every consumer can create his profile, find friends, communicate in interest groups and so on. All other social networking websites are visited rarely, however, there isn't a SN which was not known by at least a few respondents.

Analysis in terms of countries showed, that Turkish students are those who mostly use different SN. Apart from the mentioned main social networking websites, they use SN very poorly known in the other countries, such as: Twitter (Turkey PI $=0.37, \mathrm{SD}=0.45$ ), Windows 
Vincentas LAMANAUSKAS, Violeta ŠLEKIENĖ, Loreta RAGULIENĖ, Dragos IORDACHE, Costin PRIBEANU, Martin BILEK, Bulent CAVAS, Tatyana MAZUROK. Social Networking Websites From the Point of View of University Students: a Comparative Analysis

PROBLEMS OF EDUCATION IN THE $21^{\text {st }}$ CENTURY Volume 57, 2013

Live Profile (Turkey PI =0.28, SD =0.35), Dalymotion (Turkey PI =0.22, SD =0.31), Foursguare (Turkey $\mathrm{PI}=0.12, \mathrm{SD}=0.29$ ). Facebook is the most popular among Turkish respondents $(\mathrm{PI}=0.95, \mathrm{SD}=0.16)$, and respondents of Ukraine more favourably participate in YouTube $(\mathrm{PI}=0.64, \mathrm{SD}=0.37)$, than in Facebook $(\mathrm{PI}=0.25, \mathrm{SD}=0.32)$. Lithuanian and Romanian respondents similarly highly value both Facebook (Lithuanian $\mathrm{PI}=0.87, \mathrm{SD}=0.31$, Romanian $\mathrm{PI}=0.85, \mathrm{SD}=0.31$ ), and You Tube (Lithuanian PI $=0.87, \mathrm{SD}=0.21$, Romanian PI $=0.89$, $\mathrm{SD}=022)$. Czech respondents more highly value Facebook $(\mathrm{PI}=0.85, \mathrm{SD}=0.33)$, than You Tube $(\mathrm{PI}=0.79, \mathrm{SD}=0.30)$. Google + is valued by Turkish respondents best $(\mathrm{PI}=0.73, \mathrm{SD}$ $=0.39)$, and it has the least popularity among Ukrainian respondents $(\mathrm{PI}=0.46, \mathrm{SD}=0.44)$. The other countries' Google+ PI $>0.50$. Romanian respondents a little better know LinkedIn $(\mathrm{PI}=0.12, \mathrm{SD}=0.26)$ and Windows Live Profile $(\mathrm{PI}=0.10, \mathrm{SD}=0.22)$. The respondents of each country noted not a few national SN. Especially active participants in them are Ukrainian respondents.

Table 7. Students about participation in social networking websites: usage frequency (UI - usage index, , $0 \leq \mathrm{UI} \leq 1 ; \mathrm{SD}$ - standard deviation)

\begin{tabular}{|c|c|c|c|c|c|c|}
\hline \multirow{2}{*}{$\begin{array}{l}\text { Social } \\
\text { networking } \\
\text { websites }\end{array}$} & LT & RO & UA & TR & $C Z$ & Total \\
\hline & UI/SD & UI/SD & UI/SD & UI/SD & UI/SD & UI/SD \\
\hline Facebook & $0.87 / 0.31$ & $0.85 / 0.31$ & $0.25 / 0.32$ & $0.95 / 0.16$ & $0.85 / 0.33$ & $0.82 / 0.35$ \\
\hline Twitter & $0.03 / 0.14$ & $0.06 / 0.17$ & $0.08 / 0.24$ & $0.37 / 0.45$ & $0.06 / 0.21$ & $0.07 / 022$ \\
\hline Orkut & $0.002 / 0.02$ & $0.003 / 0.03$ & $0.004 / 0.03$ & $0.00 / 0.00$ & $0.004 / 0.05$ & $0.003 / 0.04$ \\
\hline My Space & $0.02 / 0.11$ & $0.03 / 0.13$ & $0.02 / 0.09$ & $0.05 / 0.18$ & $0.02 / 0.10$ & $0.02 / 0.11$ \\
\hline Linkedln & $0.006 / 0.06$ & $0.12 / 0.26$ & $0.01 / 0.08$ & $0.02 / 0.11$ & $0.03 / 0.15$ & $0.03 / 0.13$ \\
\hline $\begin{array}{l}\text { Windows } \\
\text { Live Profile }\end{array}$ & $0.03 / 0.14$ & $0.10 / 0.22$ & $0.02 / 0.11$ & $0.28 / 0.35$ & $0.03 / 0.14$ & $0.05 / 0.18$ \\
\hline $\mathrm{Hi} 5$ & $0.006 / 0.07$ & $0.05 / 0.18$ & $0.002 / 0.02$ & $0.006 / 0.04$ & $0.003 / 0.04$ & $0.009 / 0.07$ \\
\hline Bebo & $0.001 / 0.02$ & $0.01 / 0.08$ & $0.002 / 0.02$ & $0.002 / 0.02$ & $0.0014 / 0.03$ & $0.002 / 0.03$ \\
\hline OpenID & $0.004 / 0.05$ & $0.04 / 0.18$ & $0.005 / 0.05$ & $0.002 / 0.02$ & $0.02 / 0.13$ & $0.014 / 0.10$ \\
\hline YouTube & $0.87 / 0.21$ & $0.89 / 022$ & $0.64 / 0.37$ & $0.79 / 030$ & $0.79 / 0.30$ & $0.82 / 0.27$ \\
\hline Flickr & $0.027 / 0.14$ & $0.041 / 0.15$ & $0.011 / 0.089$ & $0.0043 / 0.046$ & $0.012 / 0.082$ & $0.021 / 0.12$ \\
\hline Reddit & $0.017 / 0.12$ & $0.0015 / 0.019$ & $0.019 / 0.12$ & $0.000 / 0.000$ & $0.0035 / 0.051$ & $0.010 / 0.089$ \\
\hline $\begin{array}{l}\text { Stumble- } \\
\text { Upon }\end{array}$ & $0.0036 / 0.051$ & $0.025 / 0.14$ & $0.0018 / 0.021$ & $0.0085 / 0.073$ & $0.0035 / 0.51$ & $0.0055 / 0.064$ \\
\hline Delicious & $0.0041 / 0.054$ & $0.010 / 0.073$ & $0.0035 / 0.030$ & $0.0021 / 0.023$ & $0.0 .0028 / 0.030$ & $0.0040 / 0.046$ \\
\hline Digg & $0.0033 / 0.047$ & $0.0015 / 0.019$ & $0.0018 / 0.021$ & $0.0021 / 0.023$ & $0.0011 / 0.021$ & $0.0022 / 0.035$ \\
\hline Friendster & $0.0030 / 0.039$ & $0.020 / 0.11$ & $0.0035 / 0.042$ & $0.0043 / 0.033$ & $0.0021 / 0.042$ & $0.0043 / 0.050$ \\
\hline Dailymotion & $0.026 / 0.13$ & $0.041 / 0.15$ & $0.0053 / 0.047$ & $0.22 / 0.31$ & $0.010 / 0.075$ & $0.031 / 0.14$ \\
\hline Arto & $0.0030 / 0.043$ & $0.000 / 0.000$ & $0.0018 / 0.021$ & $0.000 / 0.000$ & $0.0018 / 0.028$ & $0.0021 / 0.034$ \\
\hline Skyrock & $0.0033 / 0.052$ & $0.0015 / 0.019$ & $0.0053 / 0.036$ & $0.0043 / 0.046$ & $0.0021 / 0.035$ & $0.0029 / 0.043$ \\
\hline Tuenti & $0.0008 / 0.018$ & $0.000 / 0.000$ & $0.0018 / 0.021$ & $0.000 / 0.000$ & $0.0007 / 0.013$ & $0.0007 / 0.016$ \\
\hline Foursquare & $0.0019 / 0.032$ & $0.025 / 0.12$ & $0.0053 / 0.047$ & $0.12 / 0.29$ & $0.017 / 0.12$ & $0.016 / 0.11$ \\
\hline Vimeo & $0.049 / 0.18$ & $0.065 / 0.19$ & $0.046 / 0.15$ & $0.028 / 0.14$ & $0.033 / 0.15$ & $0.043 / 0.17$ \\
\hline Google+ & $0.58 / 0.45$ & $0.57 / 0.45$ & $0.46 / 0.44$ & $0.73 / 0.39$ & $0.53 / 0.43$ & $0.56 / 0.44$ \\
\hline
\end{tabular}


Vincentas LAMANAUSKAS, Violeta ŠLEKIENĖ, Loreta RAGULIENĖ, Dragos IORDACHE, Costin PRIBEANU, Martin BILEK, Bulent CAVAS, Tatyana MAZUROK. Social Networking Websites From the Point of View of University Students: a Comparative Analysis

The respondents were asked to evaluate social networking website functions according to their importance for them. 14 functions were presented and they had to be evaluated using five range scale from completely not important to very important. Popularity index was calculated (table 8).

Table 8. Social networking website function importance PI - popularity index, $0 \leq$ $\mathrm{PI} \leq 1 ; \mathrm{SD}$ - standard deviation

\begin{tabular}{|c|c|c|c|c|c|c|}
\hline \multirow{2}{*}{ Functions } & LT & RO & UA & TR & CZ & Total \\
\hline & $\mathrm{PI} / \mathrm{SD}$ & $\mathrm{PI} / \mathrm{SD}$ & $\mathrm{PI} / \mathrm{SD}$ & $\mathrm{PI} / \mathrm{SD}$ & $\mathrm{PI} / \mathrm{SD}$ & $\mathrm{PI} / \mathrm{SD}$ \\
\hline $\begin{array}{l}\text { 1. Communication (inclu- } \\
\text { ding communication in } \\
\text { interest groups) }\end{array}$ & $0.76 / 029$ & $0.72 / 0.29$ & $0.59 / 035$ & $0.85 / 0.17$ & $0.72 / 0.34$ & $0.74 / 0.31$ \\
\hline $\begin{array}{l}\text { 2. Learning and exchang- } \\
\text { ing information }\end{array}$ & $0.74 / 0.32$ & $0.74 / 0.44$ & $0.70 / 0.29$ & $0.84 / 0.22$ & $0.72 / 0.31$ & $0.74 / 0.32$ \\
\hline 3. Texting & $0.62 / 0.33$ & $0.50 / 0.32$ & $0.57 / 0.38$ & $0.60 / 0.27$ & $0.52 / 0.33$ & $0.57 / 0.33$ \\
\hline $\begin{array}{l}\text { 4. Exchanging photos, } \\
\text { videos and so on }\end{array}$ & $0.57 / 033$ & $0.56 / 0.30$ & $0.56 / 036$ & $0.69 / 025$ & $0.49 / 0.31$ & $0.55 / 0.32$ \\
\hline 5. Spending leisure time & $0.53 / 0.31$ & $0.49 / 031$ & $0.43 / 034$ & $0.43 / 032$ & $0.36 / 030$ & $0.46 / 0.32$ \\
\hline 6. Friend search & $0.51 / 0.34$ & $0.53 / 033$ & $0.47 / 032$ & $0.53 / 0.31$ & $0.37 / 030$ & $0.46 / 0.33$ \\
\hline $\begin{array}{l}\text { 7. Link posting from } \\
\text { internet }\end{array}$ & $0.40 / 0.34$ & $0.45 / 0.32$ & $0.32 / 0.35$ & $0.59 / 027$ & $0.60 / 0.35$ & $0.48 / 0.35$ \\
\hline $\begin{array}{l}\text { 8. Video conversations in } \\
\text { groups }\end{array}$ & $0.30 / 0.34$ & $0.36 / 0.32$ & $0.24 / 0.33$ & - & $0.28 / 0.33$ & $0.29 / 0.34$ \\
\hline 9. Flirting & $0.28 / 0.33$ & $0.25 / 0.30$ & $0.28 / 0.33$ & $0.16 / 0.26$ & $0.22 / 0.32$ & $0.25 / 0.32$ \\
\hline 10."Spying" & $0.26 / 0.33$ & $0.35 / 0.32$ & $0.25 / 0.34$ & $0.37 / 0.32$ & $0.16 / 0.30$ & $0.24 / 0.33$ \\
\hline 11. Advertisement & $0.24 / 0.31$ & $0.49 / 0.35$ & $0.23 / 0.33$ & $0.51 / 0.32$ & $0.17 / 0.30$ & $0.25 / 0.33$ \\
\hline $\begin{array}{l}\text { 12. Link posting using } \\
\text { mobile phone }\end{array}$ & $0.23 / 0.30$ & $0.34 / 0.33$ & $0.24 / 0.34$ & $0.50 / 030$ & $0.55 / 0.38$ & $0.36 / 0.37$ \\
\hline 13. Games (on-line) & $0.22 / 030$ & $0.25 / 029$ & $0.24 / 0.33$ & $0.32 / 026$ & $0.20 / 0.30$ & $0.22 / 0.30$ \\
\hline $\begin{array}{l}\text { 14. Evaluation or "Read. } \\
\text { Watch.Listen" technology }\end{array}$ & $0.21 / 0.30$ & $0.35 / 0.32$ & $0.40 / 0.36$ & $0.59 / 0.28$ & $0.20 / 0.32$ & $0.25 / 0.33$ \\
\hline
\end{tabular}

The most important fnctions for all country respondents are communication $(\mathrm{PI}=0.74$, $\mathrm{SD}=0.32$ ) learning and exchanging information $(\mathrm{PI}=0.74, \mathrm{SD}=0.32)$. Less popular is texting $(\mathrm{PI}=0.57, \mathrm{SD}=0.33)$, exchanging photos, videos and so on $(\mathrm{PI}=0.55, \mathrm{SD}=0.32)$, link posting from the internet $(\mathrm{PI}=0.48, \mathrm{SD}=0.35)$, spending leisure time in the internet $(\mathrm{PI}=0.46$, $\mathrm{SD}=0.32)$ and friend search $(\mathrm{PI}=0.46, \mathrm{SD}=0.33)$. The lowest (negative) evaluation get the games (on-line) $(\mathrm{PI}=0.22, \mathrm{SD}=0.30)$, advertisement $(\mathrm{PI}=0.25, \mathrm{SD}=0.33)$, flirting in social networking websites $(\mathrm{PI}=0.25, \mathrm{SD}=0.32)$.

Comparing SN function evaluation of separate countries, Communication (including communication in interest groups) is the most popular among Turkish respondents (PI $=0.85$, $\mathrm{SD}=0.17)$, and the least popular among the Ukrainian ones. $(\mathrm{PI}=0.59, \mathrm{SD}=0.35)$. Turkish students pay great attention to learning and exchanging information in social networking websites $(\mathrm{PI}=0.84, \mathrm{SD}=0.22)$, though the respondents of other countries pay quite a big attention to 
Vincentas LAMANAUSKAS, Violeta ŠLEKIENĖ, Loreta RAGULIENĖ, Dragos IORDACHE, Costin PRIBEANU, Martin BILEK, Bulent CAVAS, Tatyana MAZUROK. Social Networking Websites From the Point of View of University Students: a Comparative Analysis

PROBLEMS

OF EDUCATION

IN THE $21^{\text {st }}$ CENTURY

Volume 57,2013

that function as well: Lithuania ( $\mathrm{PI}=0.74, \mathrm{SD}=0.32)$, Romania( $\mathrm{PI}=0.74, \mathrm{SD}=0.44)$, Czech Republic ( $\mathrm{PI}=0.72, \mathrm{SD}=0.31)$, Ukraine $(\mathrm{PI}=0.70, \mathrm{SD}=0.29)$. Czech respondents are the least interested in friend search $(\mathrm{PI}=0.37, \mathrm{SD}=0.30)$, and Turkish and Romanian representatives are mostly interested in advertisement. Turkey $(\mathrm{PI}=0.51, \mathrm{SD}=0.32)$ and Romania $(\mathrm{PI}=0.49$, $\mathrm{SD}=0.35)$. Advertisement function for Czech representatives is the least important $(\mathrm{PI}=0.17$, $\mathrm{SD}=0.30$ ). Evaluation or "Read.Watch.Listen" technology is the most important for Turkish respondents $(\mathrm{PI}=0.59, \mathrm{SD}=0.28$ ), the youth of other countries are interested in it less.

The consumers' actions in social networking websites are public, unless the consumer indicates privacy levels (show to everybody, to friends or only to himself). Therefore, the respondents were asked whether they know, how social networking websites use published personal and other type of information. The answers showed, that the respondents of all countries involved in the research, only partly $(\mathrm{PI}=0.52, \mathrm{SD}=0.33)$ know about information usage (table $9)$. The biggest popularity index is of Czech Republic $(\mathrm{PI}=0.55, \mathrm{SD}=0.31)$, and the smallest one - of Ukraine $(\mathrm{PI}=0.48, \mathrm{SD}=0.33)$.

Table 9. The level of knowledge about how SNW use published personal or other type of information. (PI - popularity index, $0 \leq$ PI $\leq 1$; SD - standard deviation)

\begin{tabular}{|c|c|c|c|c|c|c|}
\hline $\begin{array}{l}\text { Do You know how social } \\
\text { networking websites use pub- } \\
\text { lished personal or other type of } \\
\text { information? }\end{array}$ & $\begin{array}{l}\text { LT } \\
\mathrm{PI} / \mathrm{SD}\end{array}$ & $\begin{array}{l}\text { RO } \\
\mathrm{PI} / \mathrm{SD}\end{array}$ & $\begin{array}{l}\text { UA } \\
\mathrm{PI} / \mathrm{SD}\end{array}$ & $\begin{array}{l}\text { TR } \\
\mathrm{PI} / \mathrm{SD}\end{array}$ & $\begin{array}{l}\mathrm{CZ} \\
\mathrm{PI} / \mathrm{SD}\end{array}$ & $\begin{array}{l}\text { Total } \\
\mathrm{PI} / \mathrm{SD}\end{array}$ \\
\hline & $0.51 / 0.34$ & $0.52 / 0.32$ & $0.48 / 0.33$ & $0.53 / 0.33$ & $0.55 / 0.31$ & $0.52 / 0.33$ \\
\hline
\end{tabular}

Opinion distribution of this question around the mean value is depicted in Figure 2. It can be seen, that the smallest spread is of Czech and Lithuanian respondent opinion. Turkish respondent opinion spread is the biggest of all. Opinion variance between Czech and Ukraine respondents is also not a small one. 


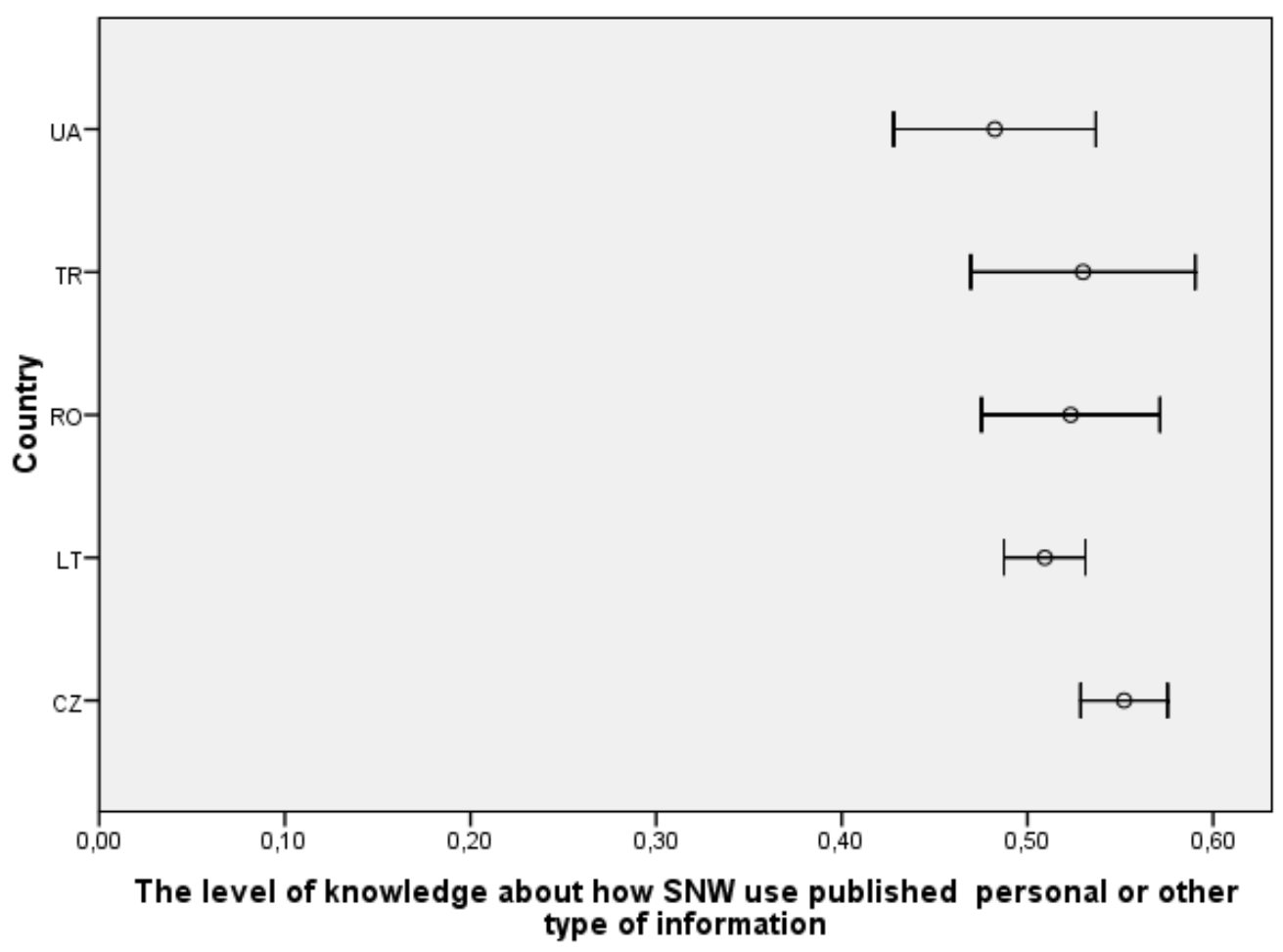

Error Bars: $95 \% \mathrm{Cl}$

Figure 2: Knowledge about SNW published information usage.

The respondents were asked to express their opinion about people who use social networking websites. Four statements were formulated and respondents were asked to evaluate them (table 10). The statement - enjoying communication $(\mathrm{PI}=0.78, \mathrm{SD}=0.20)$ has the most positive position. The highest $\mathrm{PI}$ is of Czech respondents $(\mathrm{PI}=0.84, \mathrm{SD}=0.16)$, the lowest - of Ukraine $(\mathrm{PI}=0.60, \mathrm{SD}=0.26)$. Further, people are indicated who are searching for novelties $(\mathrm{PI}=0.74, \mathrm{SD}=0.21)$, enjoying new technologies $(\mathrm{PI}=0.69, \mathrm{SD}=0.22)$. The statement that these are people, having communication difficulties in real life ( $\mathrm{PI}=0.62, \mathrm{SD}=0.28$ ) has the least positive view. Ukrainian respondents gave a very low evaluation of this $(\mathrm{PI}=0.43, \mathrm{SD}=$ $0.31)$.

Table 10. Students about people tending to use social networking websites. (PI - popularity index, $0 \leq \mathrm{PI} \leq 1 ; \mathrm{SD}$ - standard deviation)

\begin{tabular}{lllllll}
\hline $\begin{array}{l}\text { Evaluate these statements } \\
\text { about people, tending to use } \\
\text { social networks }\end{array}$ & $\mathrm{LT}$ & $\mathrm{RO}$ & $\mathrm{UA}$ & $\mathrm{TR}$ & $\mathrm{CZ}$ & Total \\
\cline { 2 - 7 } & $\mathrm{PI} / \mathrm{SD}$ & $\mathrm{PI} / \mathrm{SD}$ & $\mathrm{PI} / \mathrm{SD}$ & $\mathrm{PI} / \mathrm{SD}$ & $\mathrm{PI} / \mathrm{SD}$ & $\mathrm{PI} / \mathrm{SD}$ \\
\hline 1. Enjoying communication & $0.78 / 0.19$ & $0.80 / 0.19$ & $0.60 / 0.26$ & $0.84 / 0.16$ & $0.78 / 0.20$ \\
\hline 2. Enjoying new technologies & $0.71 / 0.21$ & $0.79 / 0.18$ & $0.58 / 0.25$ & $0.66 / 0.22$ & $0.69 / 0.22$ \\
\hline 3. Searching for novelties & $0.71 / 0.22$ & $0.64 / 0.20$ & $0.70 / 0.26$ & - & $0.80 / 0.18$ & $0.74 / 0.21$ \\
\hline $\begin{array}{l}\text { 4. Having communication dif- } \\
\text { ficulties in real life }\end{array}$ & $0.63 / 0.27$ & $0.55 / 0.28$ & $0.43 / 0.31$ & $0.66 / 0.27$ & $0.62 / 0.28$ \\
\hline
\end{tabular}


Vincentas LAMANAUSKAS, Violeta ŠLEKIENĖ, Loreta RAGULIENĖ, Dragos IORDACHE, Costin PRIBEANU, Martin BILEK, Bulent CAVAS, Tatyana MAZUROK. Social Networking Websites From the Point of View of University Students: a Comparative Analysis

PROBLEMS

OF EDUCATION

IN THE $21^{\text {st }}$ CENTURY

Volume 57, 2013

The respondents were asked, whether participation in social networking websites improves their information communication abilities. They could answer: yes, it improves; partly improves; has no influence. The respondents' analysis of the countries involved in the research, showed (table11), that the most positive evaluation of the relationship improvement between participation in the social networks and information communication abilities was of Turkish respondents $(\mathrm{PI}=0.51, \mathrm{SD}=0.39)$. The slightest relationship between $\mathrm{SN}$ and information abilities was noticed by Czech respondents $(\mathrm{PI}=0.30, \mathrm{SD}=0.28)$. It can be seen, that respondents not very highly value that $\mathrm{SN}$ improve their information communication abilities.

Table 11. Students' opinion about SN and information communication abilities (PI - popularity index, $0 \leq \mathrm{PI} \leq 1$; SD - standard deviation)

\begin{tabular}{lllllll}
\hline $\begin{array}{l}\text { Does the usage of SN } \\
\text { websites improve Your } \\
\text { information communica- } \\
\text { tion abilities? }\end{array}$ & $\begin{array}{l}\text { LT } \\
\text { PI/SD }\end{array}$ & $\begin{array}{l}\text { RO } \\
\text { PI/SD }\end{array}$ & $\begin{array}{l}\text { UA } \\
\text { PI/SD }\end{array}$ & $\begin{array}{l}\text { TR } \\
\text { PI/SD }\end{array}$ & $\begin{array}{l}\text { CZ } \\
\text { PI/SD }\end{array}$ & $\begin{array}{l}\text { Total } \\
\text { PI/SD }\end{array}$ \\
\hline & $0.38 / 0.31$ & $0.40 / 0.32$ & $0.43 / 0.35$ & $0.51 / 0.39$ & $0.30 / 0.28$ & $0.36 / 0.32$ \\
\hline
\end{tabular}

Opinion distribution of this question around the mean value analysis (Figure 3) shows, that the smallest spread is of Czech and Lithuanian respondent opinion, though their positive views are not high (Czech - PI $=0.30, \mathrm{SD}=0.28$; Lithuanian - PI $=0.38, \mathrm{SD}=0.31)$. And Turkish respondents' opinion distribution is the biggest, however, their PI value is the highest of all. Not a small opinion variance is noticed between Czech and Ukraine respondents.

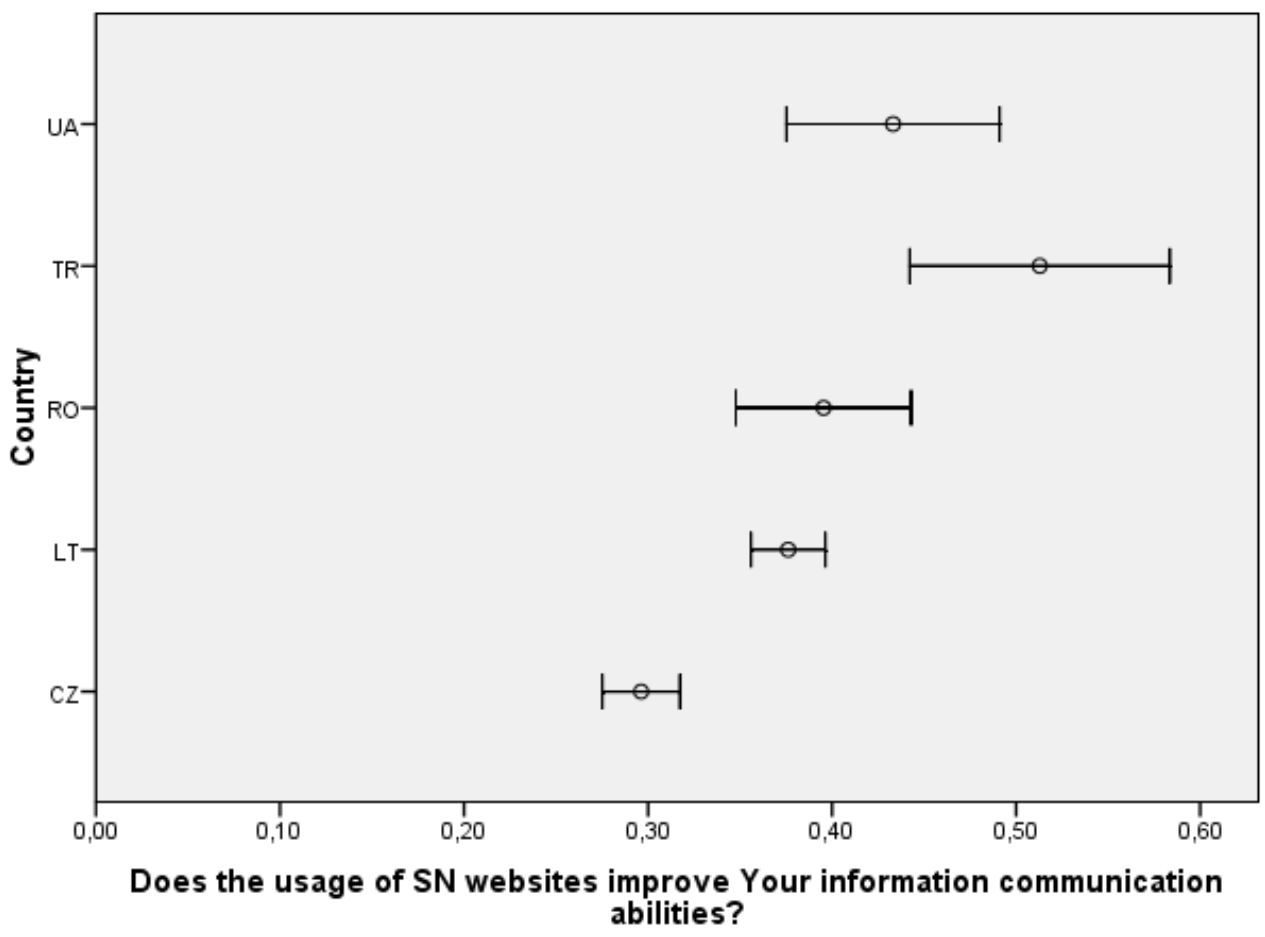

Error Bars: $95 \% \mathrm{Cl}$

Figure 3: SNW and consumers' information communication abilities. 
Vincentas LAMANAUSKAS, Violeta ŠLEKIENĖ, Loreta RAGULIENĖ, Dragos IORDACHE, Costin PRIBEANU, Martin BILEK, Bulent CAVAS, Tatyana MAZUROK. Social Networking Websites From the Point of View of University Students: a Comparative Analysis

Social networking websites reveal the participants' activity, sociality, because the main information is this, what the consumer wrote, what his mood was, who he started dating, who he started going out with, what photos installed, how he commentates, who he joined and so on. 26 statements about social networks were presented in the questionnaire and the respondents were asked to evaluate them in the 5 range scale from completely agree to completely disagree.

Each country respondents involved in the research rather highly value social networks in various aspects and the majority of all country evaluations are similar (fluctuate at about average) (table 12). Almost all people of every country, having participated in the questionnaire, use social networks and accentuate that $\mathrm{SN}$ is a perfect means of communication $(\mathrm{PI}=0.74, \mathrm{SD}$ $=0.22)$ and is a good possibility to find out various news $(\mathrm{PI}=0.74, \mathrm{SD}=0.22)$. However, at the same time they emphasize, that communication in virtual space will never substitute direct peoples 'relations $(\mathrm{PI}=0.88, \mathrm{SD}=0.24)$, that most people belonging to $S N$ websites don 't know, who they share information with about themselves, their hobbies and life $(\mathrm{PI}=0.71, \mathrm{SD}$ $=0.23$ ) and, that information conveyed by $S N$ websites can have negative influence on teenagers' behaviour and health $(\mathrm{PI}=0.71, \mathrm{SD}=0.21)$. A little less worry about communication in virtual space $(\mathrm{PI}=0.78, \mathrm{SD}=0.24)$ and about $\mathrm{SN}$ negative influence on teenagers' behaviour and health $(\mathrm{PI}=0.67, \mathrm{SD}=0.21)$ expressed respondents from Romania; and about not having knowledge about personal information spread - respondents from Ukraine $(\mathrm{PI}=0.65, \mathrm{SD}=$ 0.21 ). These are five out of presented 26 most significantly expressed, contrary and supplementing one another statements about SN.

Table 12. Students' opinion about social networking websites. (PI - popularity index, $0 \leq \mathrm{PI} \leq 1 ; \mathrm{SD}$ - standard deviation)

\begin{tabular}{lllllll}
\hline $\begin{array}{l}\text { Evaluate these statements } \\
\text { about social networking web- } \\
\text { sites (SN websites) }\end{array}$ & LT & RO & UA & TR & CZ & Total \\
\cline { 2 - 6 } & PI/SD & PI/SD & PI/SD & PI/SD & PI/SD & PI/SD \\
\hline $\begin{array}{l}\text { 1. SN websites distract people } \\
\text { from reality }\end{array}$ & $0.65 / 0.24$ & $0.69 / 0.24$ & $0.71 / 0.26$ & $0.66 / 0.26$ & $0.68 / 0.24$ & $0.67 / 0.24$ \\
\hline $\begin{array}{l}\text { 2. SN websites earn a lot of } \\
\text { money using free personal }\end{array}$ & $0.63 / 0.21$ & $0.74 / 0.21$ & $0.59 / 0.23$ & $0.64 / 0.23$ & $0.58 / 0.21$ & $0.62 / 0.22$ \\
information & & & & & & \\
\hline $\begin{array}{l}\text { 3. People of lower education } \\
\text { mostly use SN websites }\end{array}$ & $0.38 / 0.23$ & $0.51 / 0.26$ & $0.42 / 0.25$ & $0.39 / 0.28$ & $0.28 / 0.21$ & $0.36 / 0.24$ \\
\hline $\begin{array}{l}\text { 4. People taking part in SN } \\
\text { websites contribute to ICT } \\
\text { development }\end{array}$ & $0.58 / 0.20$ & $0.59 / 0.19$ & $0.48 / 0.25$ & $0.62 / 0.25$ & $0.53 / 0.19$ & $0.56 / 0.21$ \\
\hline $\begin{array}{l}\text { 5. SN websites help to make } \\
\text { friends }\end{array}$ & $0.71 / 0.20$ & $0.66 / 0.23$ & $0.65 / 0.23$ & $0.63 / 0.26$ & $0.61 / 0.24$ & $0.67 / 0.23$ \\
\hline $\begin{array}{l}\text { 6. SN websites is a perfect } \\
\text { means of communication }\end{array}$ & $0.76 / 0.19$ & $0.72 / 0.22$ & $0.69 / 0.23$ & $0.62 / 0.27$ & $0.74 / 0.23$ & $0.74 / 0.22$ \\
\hline $\begin{array}{l}\text { 7. SN websites can encourage } \\
\text { the youth for suicide }\end{array}$ & $0.53 / 0.25$ & $0.46 / 0.26$ & $0.47 / 0.30$ & $0.51 / 0.27$ & $0.58 / 0.24$ & $0.54 / 0.26$ \\
\hline $\begin{array}{l}\text { 8. SN websites dehumanise } \\
\text { society }\end{array}$ & $0.55 / 0.23$ & $0.55 / 0.25$ & $0.50 / 0.25$ & $0.44 / 0.26$ & $0.64 / 0.25$ & $0.57 / 0.25$ \\
\hline $\begin{array}{l}\text { 9. SN websites encourage people } \\
\text { estrangement }\end{array}$ & $0.60 / 0.25$ & $0.57 / 0.22$ & $0.53 / 0.22$ & $0.59 / 0.29$ & $0.65 / 0.27$ & $0.61 / 0.26$ \\
\hline $\begin{array}{l}\text { 10. Staying in SN websites is } \\
\text { more fashionable than useful }\end{array}$ & $0.66 / 0.25$ & $0.66 / 0.24$ & $0.56 / 0.25$ & $0.75 / 0.24$ & $0.53 / 0.29$ & $0.61 / 0.27$ \\
\hline & & & & & & \\
\hline
\end{tabular}

$\mid \begin{aligned} & \text { PROBLEMS } \\ & \text { OF EDUCATION } \\ & \text { IN THE 21 } 1^{\text {st }} \text { CENTURY } \\ & \text { Volume 57, } 2013\end{aligned}$ 
Vincentas LAMANAUSKAS, Violeta ŠLEKIENĖ, Loreta RAGULIENĖ, Dragos IORDACHE, Costin PRIBEANU, Martin BILEK, Bulent CAVAS, Tatyana MAZUROK. Social Networking Websites From the Point of View of University Students: a Comparative Analysis

OF EDUCATION

IN THE $21^{\text {st }}$ CENTURY Volume 57, 2013

\begin{tabular}{lcccccc}
\hline $\begin{array}{l}\text { 11. Communication in virtual } \\
\text { space gives bigger opportunities }\end{array}$ & $0.61 / 0.22$ & $0.59 / 0.22$ & $0.43 / 0.27$ & $0.58 / 0.26$ & $0.56 / 0.27$ & $0.57 / 0.25$ \\
\hline $\begin{array}{l}\text { 12. Constant staying in virtual } \\
\text { environment causes damage to } \\
\text { person's socialization }\end{array}$ & $0.67 / 0.24$ & $0.67 / 0.25$ & $0.63 / 0.24$ & $0.77 / 0.23$ & $0.74 / 0.23$ & $0.70 / 0.24$ \\
\hline $\begin{array}{l}\text { 13. Communication in virtual } \\
\text { space will never substitute direct } \\
\text { peoples' relations }\end{array}$ & $0.89 / 0.17$ & $0.78 / 0.24$ & $0.85 / 0.22$ & $0.83 / 0.23$ & $0.89 / 0.18$ & $0.88 / 0.19$ \\
\hline
\end{tabular}

\begin{tabular}{lllllll}
\hline $\begin{array}{l}\text { 14. Most people belonging to SN } \\
\text { websites don't know, who they } \\
\text { share information with about } \\
\text { themselves, their hobbies and life }\end{array}$ & $0.72 / 0.23$ & $0.70 / 0.22$ & $0.65 / 0.21$ & $0.74 / 0.22$ & $0.71 / 0.23$ & $0.71 / 0.23$ \\
\hline $\begin{array}{l}15 . \text { SN websites is a reliable } \\
\text { computer device }\end{array}$ & $0.45 / 0.22$ & $0.60 / 0.20$ & $0.44 / 0.20$ & $0.39 / 0.36$ & $0.41 / 0.21$ & $0.44 / 0.22$ \\
\hline $\begin{array}{l}\text { 16. Information conveyed by } \\
\begin{array}{l}\text { SN websites can have negative } \\
\text { influence on teenagers' behaviour } \\
\text { and health }\end{array}\end{array}$ & $0.72 / 0.21$ & $0.67 / 0.21$ & $0.68 / 0.23$ & $0.69 / 0.19$ & $0.73 / 0.21$ & $0.71 / 0.21$ \\
\hline
\end{tabular}

\begin{tabular}{lllllll}
$\begin{array}{l}\text { 17. SN websites make negative } \\
\text { influence on pupils' marks and } \\
\text { ability to concentrate }\end{array}$ & $0.73 / 0.21$ & $0.67 / 0.23$ & $0.65 / 0.26$ & $0.71 / 0.20$ & $0.69 / 0.22$ & $0.70 / 0.22$ \\
\hline $\begin{array}{l}\text { 18. One can find a lot of useful } \\
\text { information in SN websites }\end{array}$ & $0.67 / 0.23$ & $0.72 / 0.19$ & $0.67 / 0.20$ & $0.71 / 0.20$ & $0.74 / 0.22$ & $0.70 / 0.22$ \\
\hline $\begin{array}{l}\text { 19. SN websites is a good means } \\
\text { of spending your leisure time }\end{array}$ & $0.62 / 0.26$ & $0.60 / 0.25$ & $0.50 / 0.27$ & $0.52 / 0.26$ & $0.31 / 0.25$ & $0.50 / 0.29$ \\
\hline $\begin{array}{l}\text { 20. SN websites is good possibil- } \\
\text { ity to find out various news }\end{array}$ & $0.75 / 0.21$ & $0.76 / 0.21$ & $0.71 / 0.23$ & $0.75 / 0.20$ & $0.67 / 0.20$ & $0.72 / 0.21$ \\
\hline $\begin{array}{l}\text { 21. SN websites is a good thing } \\
\text { for those, who don't perceive } \\
\text { what privacy is }\end{array}$ & $0.61 / 0.25$ & $0.58 / 0.26$ & $0.50 / 0.22$ & $0.51 / 0.26$ & $0.62 / 0.23$ & $0.60 / 0.25$ \\
\hline
\end{tabular}

22. This is a good opportunity to learn, improve communicate with the whole world without going out from home

\begin{tabular}{lllllll}
\hline $\begin{array}{l}\text { 23. SN websites is a wonderful } \\
\text { means of relaxation }\end{array}$ & $0.60 / 0.25$ & $0.65 / 0.23$ & $0.54 / 0.25$ & $0.52 / 0.27$ & $0.49 / 0.26$ & $0.56 / 0.26$ \\
\hline $\begin{array}{l}\text { 24. SN websites provide nation- } \\
\text { al and international knowledge }\end{array}$ & $0.63 / 0.23$ & $0.68 / 0.20$ & $0.40 / 0.26$ & $0.70 / 0.22$ & $0.65 / 0.23$ & $0.63 / 0.24$ \\
\hline $\begin{array}{l}\text { 25. SN websites is a perfect } \\
\text { means for self-advertisement }\end{array}$ & $0.73 / 0.22$ & $0.69 / 0.21$ & $0.64 / 0.22$ & $0.67 / 0.23$ & $0.50 / 0.27$ & $0.64 / 0.26$ \\
\hline $\begin{array}{l}\text { 26. SN websites save time, allow } \\
\text { acting more effectively }\end{array}$ & $0.56 / 0.26$ & $0.58 / 0.22$ & $0.42 / 0.29$ & $0.46 / 0.28$ & $0.44 / 0.28$ & $0.51 / 0.28$ \\
\hline
\end{tabular}


Vincentas LAMANAUSKAS, Violeta ŠLEKIENĖ, Loreta RAGULIENĖ, Dragos IORDACHE, Costin PRIBEANU, Martin BILEK, Bulent CAVAS, Tatyana MAZUROK. Social Networking Websites From the Point of View of University Students: a Comparative Analysis

Here, also are the statements rather highly evaluated by all countries, expressing positi- 75 ve and negative attitude towards SNW:

- One can find a lot of useful information in $S N$ websites (PI = 0.70, SD =0.22), SN websites help to make friends $(\mathrm{PI}=0.67, \mathrm{SD}=0.23)$, SN websites is a perfect means for self-advertisement $(\mathrm{PI}=0.64, \mathrm{SD}=0.26)$ (though Ukrainian representatives doubt about this $(\mathrm{PI}=0.50, \mathrm{SD}=0.27)$.

- Constant staying in virtual environment causes damage to person's socialization $(\mathrm{PI}=0.70, \mathrm{SD}=0.24), S N$ websites distract people from reality $S T(\mathrm{PI}=0.67, \mathrm{SD}=$ 0.24), SN websites earn a lot of money using free personal information (PI $=0.62$, $\mathrm{SD}=0.22)$, Staying in $S N$ websites is more fashionable than useful (PI =0.61, SD $=0.27)$, SN websites encourage people estrangement $(\mathrm{PI}=0.61, \mathrm{SD}=0.26)$.

This shows, that SN evaluation is not and cannot be unambiguous, that social networks are a multidimentional construct, covering both positive and negative influence on personality and her socialization.

Analysis, regarding countries, having participated in the research, showed, that Lithuanian $(\mathrm{PI}=0.62, \mathrm{SD}=0.26)$ and Romanian $(\mathrm{PI}=0.60, \mathrm{SD}=0.25)$ respondents are convinced, that $\mathrm{SN}$ is a good means of spending your leisure time. Turkish (PI $=0.52, \mathrm{SD}=0.26)$ and Ukrainian $(\mathrm{PI}=0.50, \mathrm{SD}=0.27)$ respondents doubt in this, and Czech respondents do not agree with the statement, that $S N$ is a wonderful means of relaxation $(\mathrm{PI}=0.31, \mathrm{SD}=0.25)$. The opinions of the countries also differed evaluating the statement, that $S N$ websites provide national and international knowledge. This statement got the highest evaluation by Turkish respondents $(\mathrm{PI}=0.70, \mathrm{SD}=0.22)$. Romanian $(\mathrm{PI}=0.68, \mathrm{SD}=0.20)$, Czech $(\mathrm{PI}=0.65, \mathrm{SD}=0.23)$ and Lithuanian $(\mathrm{PI}=0.63, \mathrm{SD}=0.23)$ respondents have no doubt in this as well. However, Ukrainian representatives expressed negative position in this aspect $(\mathrm{PI}=0.40, \mathrm{SD}=0.26)$. They doubt and do not agree, that SN websites provide national and international knowledge. Romanian representatives state, that $\mathrm{SN}$ websites is a reliable computer device $(\mathrm{PI}=0.60, \mathrm{SD}=0.20)$, though respondents of other countries doubt in this or do not agree $(\mathrm{PI}=0.44, \mathrm{SD}=0.22)$. A similar differenciation of opinions is noticed of the statement People of lower education mostly use SN. Only Romanian respondents expressed doubt in this statement (PI $=0.51, \mathrm{SD}=0.26)$. The other countries' (LT, UA, TR, CZ) representatives have negative attitude in this aspect (PI $=0.36, \mathrm{SD}=0.24)$. They are convinced, that SN consumers are not of a lower intellect.

It is obvious, that SN both positive and negative influence on every personality and on all society is not an unambiguous phenomenon. Therefore, its evaluation cannot be unambiguous as well. It is determined by various reasons, which need to be differentiated and researched more exhaustively.

\section{Conclusions}

It has been stated, that the majority of respondents of all countries involved in the research use the internet a few times a day. Also, more than half of them visit social networking websites a few times a day, and even three quarters of Ukrainian respondents do this. It is obvious, that using the internet and visiting social networking websites are closely and directly related things.

Social networking websites are popular among the youth of the countries, having participated in the research: SNW are the most popular among Turkish respondents and the least popular among Czech ones. SN evaluations of other country respondents (LT, RO, UA) are close to average.

It has been stated, that even though respondents know quite many social networking websites, the most frequently visited and the most popular are three: You Tube, Facebook, Google+. Different SN mostly use Turkish respondents, who, without mentioned main social networking websites, participate in the other SN, poorly known in the other countries (Twitter, Windows Live Profile, Dalymotion). Every country respondents also participate in national SN. Ukraine respondents very actively participate in them. 
Vincentas LAMANAUSKAS, Violeta ŠLEKIENĖ, Loreta RAGULIENĖ, Dragos IORDACHE, Costin PRIBEANU, Martin BILEK, Bulent CAVAS, Tatyana MAZUROK. Social Networking Websites From the Point of View of University Students: a Comparative Analysis

PROBLEMS

OF EDUCATION

IN THE $21^{\text {st }}$ CENTURY Volume 57,2013

The most important SNW functions for all country respondents are communication and learning and exchanging information. Less important are: texting, spending leisure time in the internet, friend search, exchanging photos, videos and so on. Games (on-line), advertisement and flirting in SN get the lowest (negative) evaluation. Evaluation or "Read.Watch.Listen" technology is the most important for Turkish respondents. The other countries' youth are interested less in this.

It is worrying, that all country, having participated in the research, respondents only partly know how social networking websites use published personal and other type of information.

It has been stated, that all country, having participated in the research, respondents are active social network users and discern both positive and negative influence on personality and her socialization:

- One can claim, that all country, having participated in the research, respondents rather highly value social networks and acknowledge their usefulness both for communication and leisure and for social and obtaining information needs.

- Respondents are rather strongly convinced, that SNW distract people from reality, make real relationship among people weaker, encourage people estrangement, even encourage for suiside.

The analysis of the respodents' opinion about SN according to countries, having taken part in the research, showed:

- That Lithuanian and Romanian respondents are convinced, that SNW is a good means of spending leisure time. Turkish and Ukrainian respondents doubt in this, and Czech ones - do not agree with this statement.

- The statement, that SNW provide national and international knowledge, was evaluated at the highest by Turkish respondents, Romanian, Czech and Lithuanian respondents do not doubt in this, and Ukrainian representatives doubt or do not agree.

- Romanian representatives state, that $\mathrm{SN}$ is a reliable computer device, though respondents from other countries doubt in this or do not agree.

Such not unambiguous SNW evaluation position is interpreted as positive. On the other hand, it is obvious, that educational potential of social networking websites in students ${ }^{6}$ evaluation is expressed rather weakly and it raises a grounded worry.

\section{Note}

This paper was presented at the IOSTE Eurasia Regional Symposium \& Brokerage Event Horizon 2020-EU Framework Programme for Research and Innovation, October 30-November 1, 2013, Antalya, Turkey. It was approved by the Symposium scientific committee.

\section{References}

Acquisti, A., \& Gross, R. (2005). Imagined communities: Awareness, information sharing, and privacy on the Facebook. In Proceedings of the $6^{\text {th }}$ Workshop on Privacy Enhancing Technologies, (Ed.) P. Golle \& G. Danezis, 36-58. Cambridge: Robinson College.

Awouters, V., Palmaers, D., Veltjen, A. (2011). 2Facebook or not 2Facebook in Education: that's the question? Teaching and Learning on Facebook. In.: Splet izobraževanja in raziskovanja z IKT / Enabling education and research with ICT (Mednarodna konferenca Splet izobraževanja in raziskovanja z IKT - SIRikt 2011, Kranjska Gora, 13. - 16. april 2011 / The 5th International Conference SIRikt, 13th - 16th April 2011). Ljubljana: Mi ka, p. 148-151.

Baltaretu, C. M., Balaban, D. C. (2010). Motivation in Using Social Network Sites by Romanian Students. A Qualitative Approach. Journal of Media Research, 6, 67-74.

Ellison N.B., Steinfield C., Lampe C. (2007) The Benefits of Facebook "Friends:" Social Capital and College Students' Use of Online Social Network Sites. Journal of Computer-Mediated Communication 12, 1143-1168. 
Vincentas LAMANAUSKAS, Violeta ŠLEKIENĖ, Loreta RAGULIENĖ, Dragos IORDACHE, Costin PRIBEANU, Martin BILEK, Bulent CAVAS, Tatyana MAZUROK. Social Networking Websites From the Point of View of University Students: a Comparative Analysis

Gemmill, E., \& Peterson, M. (2006). Technology use among college students: Implications for Student Affairs Professionals. NASPA Journal, 43 (2), 280-300.

Joinson, A. N. (2008). 'Looking at', 'Looking up' or 'Keeping up with' People? Motives and Uses of Facebook. In. CHI 2008 Proceedings · Online Social Networks (April 5-10, 2008). Florence, Italy, p. 1027-1036.

Jung, Y., Gray R, Lampe C., Ellison N. B. (2013) Favors from Facebook 'Unpacking dimensions of social capital. Proceedings of CHI 2013, 1143-1168, ACM.

Karliček, M., Tomek, I., Hasprova, M., Zamazalova, M. (2012). Social Networking Sites in the Czech Republic: an Empirical Study. Management \& Marketing Challenges for the Knowledge Society, $7(1), 15-28$.

Kolek, E.A. \& Saunders, D. (2008). Online disclosure: An empirical examination of undergraduate Facebook profiles. NASPA Journal, 45 (1).

Lamanauskas, V., Šlekienè, V., Ragulienè, L. (2010). Lietuvos universitetų studentų patirtis naudoti informacines komunikacines technologijas. Gamtamokslinis ugdymas / Natural Science Education, 3 (29), 14-27.

Marinescu, V. (2011). The youth of social networks uses and the social networks for youth A comparison between the uses of Facebook and HI5 among Romanian youth. Проблеми на постмодерността, Том I, Брой 2. Retrieved October 04, 2013, from http://ppm.swu.bg/media/32065/ marinescu,\%20v.\%20the\%20youth\%20of\%20social\%20networks $\% 20$ uses $\% 20$ and $\% 20$ the $\% 20$ social $\% 20$ networks $\% 20$ for $\% 20$ youth $\% 20 \mathrm{a} \% 20$ comparison $\% 20$ between $\% 20$ the $\% 20$ uses $\% 20$ of $\% 20$ facebook $\% 20$ and $\% 20$ hi5\%20among\%20romanian\%20youth.pdf

Mendez, Jesse P., Curry, J., Mwavita, M., Kennedy, K., Weinland, K., Bainbridge, K. (2009). To Friend or Not to Friend: Academic Interaction on Facebook. International Journal of Instructional Technology \& Distance Learning, 6 (9). Retrieved on 20 April 2012 from, http://www.itdl.org/ Journal/Sep_09/article03.htm

Putnam, R. D. (2000). Bowling alone: The collapse and revival of American community. New York: Simon \& Schuster.

Rouis, S., Limayem, M., Salehi-Sangari, E. (2011). Impact of Facebook Usage on Students' Academic Achievement: Role of Self-regulation and Trust. Electronic Journal of Research in Educational Psychology, 9 (3), 961-994.

Taynton, K. (2012). The Net Generation in Japan: a Survey of Internet Behaviours of Students Aged 1321. Teaching English with Technology, 12 (1), 3-19.

Turan, Z., Tinmaz, H., Goktas, Y. (2013). The Reasons for Non-Use of Social Networking Websites by University Students. Comunicar: Scientific Journal of Media Education, 21 (41), 137-145.

Ünal Tutgun A., Köroğlu, O. (2013). A comparative study of social network usage and adoption among Turkish prospective teachers. Mevlana International Journal of Education (MIJE), 3 (4), 24-42.

Advised by Laima Railiene, University of Siauliai, Lithuania

Received: September 16, 2013

Accepted: November 15, 2013 
Vincentas LAMANAUSKAS, Violeta ŠLEKIENĖ, Loreta RAGULIENĖ, Dragos IORDACHE, Costin PRIBEANU, Martin BILEK, Bulent CAVAS, Tatyana MAZUROK. Social Networking Websites From the Point of View of University Students: a Comparative Analysis

PROBLEMS

OF EDUCATION

IN THE $21^{\text {st }}$ CENTURY Volume 57,2013
Professor, Natural Science Education Research Centre, Siauliai University, 25119 P.Višinskio Street, LT- 76351, Siauliai, Lithuania.

Phone: +370 68795668

E-mail: lamanauskas@projektas.It

Website: http://www.lamanauskas.projektas.It

Violeta Šlekienė $\quad$ PhD., Associate Professor, Department of Physics, Faculty of Natural Sciences, iauliai University, 19 P. Visinskio Street, LT-77156 Siauliai, Lithuania.

Phone: +370 41595721

E-mail: fk@fm.su.It

Website: http://www.su.lt/ This e-mail address is being protected from spambots. You need JavaScript enabled to view it

Loreta Raguliené Associate Professor, Department of Physics, Faculty of Natural Sciences, Šiauliai University, 19 P. Visinskio Street, LT-77156 Siauliai, Lithuania.

Phone: +370 41595721

E-mail: fk@fm.su.It

Website: http://www.su.lt/

Dragos Daniel lordache

PhD., Researcher, National Institute for Research and Development in Informatics - ICl Bucharest, Blvd. Mareşal Averescu 8-10, 011455 Bucharest, Romania. E-mail: iordache@ici.ro

\begin{tabular}{|c|c|}
\hline Costin Pribeanu & $\begin{array}{l}\text { PhD., Senior Researcher, National Institute for Research and Development in } \\
\text { Informatics - ICI Bucharest, Blvd. Mareşal Averescu 8-10, } 011455 \text { Bucharest, } \\
\text { Romania. } \\
\text { E-mail: pribeanu@ici.ro } \\
\text { Website: http://rochi.ici.ro/pribeanu/ }\end{array}$ \\
\hline Martin Bilek & $\begin{array}{l}\text { Professor, Department of Chemistry - Section for Chemistry Didactics, Faculty } \\
\text { of Science, University of Hradec Kralove, Rokitanskeho 62, CZ-50003 Hradec } \\
\text { Kralove, Czech Republic } \\
\text { Phone: +420- 493331164. } \\
\text { E-mail: martin.bilek@uhk.cz } \\
\text { Website: http://www.uhk.cz/ }\end{array}$ \\
\hline Bulent Cavas & $\begin{array}{l}\text { PhD., Associate Professor, Dokuz Eylul University, Department of Science } \\
\text { Education, Izmir, Turkey } \\
\text { Phone: +905324267927 } \\
\text { E-mail: bulent.cavas@deu.edu.tr }\end{array}$ \\
\hline Tatyana Mazurok & $\begin{array}{l}\text { Professor, Department of Applied Mathematics and Informatics, Faculty of } \\
\text { Physics and Mathematics, South Ukrainian National Pedagogical University } \\
\text { named after K.D. Ushinsky, } 26 \text { Staroportofrankovskaya Street, 6520, Odessa, } \\
\text { Ukraine. } \\
\text { E-mail: pdpu@pdpu.edu.ua } \\
\text { Website: http://www.pdpu.edu.ua/ }\end{array}$ \\
\hline
\end{tabular}

